\title{
Current state of quantum theory
}

\begin{abstract}
Standard and path integral formulations of quantum mechanics are reviewed briefly followed by the formulations based on the gauge transformations, which are Wheeler's formulation and another one founded upon an extension of Hamilton's action principle. Various interpretations of quantum mechanics are discussed and compared. Recent developments in quantum field theory are commented on.
\end{abstract}

Keywords: quantum theory, Hamilton's action principle, Nelson's formulation, wave function
Volume I Issue 4 - 2017

SR Vatsya

648 Inverness Ave, Canada

Correspondence: Shiv R Vatsya, 648 Inverness Ave in London, ON, Canada, Tel 519474 I I83, Email raj.vatsya@gmail.com

Received:September 14, 2017| Published: October 17, 2017

\section{Introduction}

The original formulation of quantum mechanics is based on some empirically deduced postulates available in the texts. This founding premise is discomforting for a scientific discipline should be founded upon a conceptual ground and a systematic, logically consistent structure. This situation is improved by Feynman's path integral formulation ${ }^{1-4}$ but only partially. Further disconcerting situations developed consequent of the original formulation, mainly with the quantum mechanical understanding of measurement, i.e., observations. The observed impact of the method of observation on its outcome presents an enigmatic situation, which is not well understood. This problem is best illustrated by the quintessential double slit experiment. With two slits open, individual microscopic entities such as the electrons and photons, arrive on the observation screen as classical particles but not at the classically prescribed locations. As the number of arrivals increases, they cluster about certain locations and after infinitely many of them have arrived on the screen, the density distribution resembles the intensity pattern produced by two interfering wavelets emanating from the slits. Such observations underlie the doctrine of the wave-particle duality, which fuses two contradictory concepts together. If an observation behind one of the slits is made that enables an extraction of "which path" information, i.e., which path some particles took, the density distribution becomes as produced by the particles passing through both slits. If the "which path" information is erased, the distribution again becomes interference-like. Both behaviors can be observed in different parts of the data in a single experiment. ${ }^{5}$

Numbers of interpretations have been developed to address the above issue that are described in the paper and commented upon in appropriate sections. None of the interpretations developed to explain such phenomena is universally accepted; their popularity has varied with times. Despite a weak foundation, the theory has survived due to its remarkable successes in describing the observed physical phenomena. Nevertheless, the resulting issues of fundamental nature are not yet fully resolved. This review describes the formulations and the weaknesses in the foundational structure of quantum mechanics.

Parallel efforts have continued to formulate quantum mechanics in terms of the concept of gauge transformations underlying Weyl's geometry ${ }^{6}$ hoping to resolve the foundational issues. First such attempt was made by London ${ }^{7}$ but little progress was made. However, some recent developments based on this concept, have been successful in making major advances. ${ }^{8,9}$ The formulation including such developments is referred to as the geometrical formulation. This brief review describes this formulation and compares its advantages over the standard ones.

Wheeler's Wiener integral formulation in Weyl's geometry ${ }^{10}$ supplemented with Nelson's formulation ${ }^{11}$ also relates the gauge transformations with quantum mechanics but in a limited way. This development, although noteworthy, is founded upon rather untenable assumptions and it has not been found to be very useful. This formulation has been included and commented upon for its relevance and historical value. Included also is a comparative discussion of the Aharonov-Bohm effect ${ }^{12}$ that is geometrical in nature as understood in terms of the standard formulation but more so in terms of the geometrical formulation. There are troubling issues with quantum field theory also, which are not discussed here in detail but briefly commented on.

\section{Gauge transformations}

In the Riemann spaces, the length of a vector remains constant under parallel transport. The Weyl geometry ${ }^{6}$ was developed by assuming that the length $l_{x}$ of a vector undergoes a change of $\delta l_{x}=a \phi_{\mu}^{\prime} d x^{\mu} l_{x}$ under parallel transport from an arbitrary point $x$ to a neighboring point $x+d x$, where Weyl's weight $a$ is a nonzero constant and $\phi_{\mu}^{\prime}$ , termed the Weyl gauge potentials, are the components of a vector, Weyl's vector potential. It follows that the length $l_{y}$ at a point $y$ of a vector transported from $x$ to $y$ along a trajectory $\rho_{x y}$ is given by

$$
l_{y}=\exp \left[a \int_{\rho_{x y}} \phi_{\mu}^{\prime} d x^{\mu}\right] l_{x} .
$$

Also, the gauge can be assigned essentially arbitrarily at every point. This is equivalent to recalibrating the lengths by redefining the metric, $\tilde{g}(x) \rightarrow \kappa^{2}(x) \tilde{g}(x)$, which transforms the length $l_{x} \rightarrow \kappa(x) l_{x}=l_{x}^{\prime}$. It follows from (1) that as a result of this recaliberation,

$$
l_{y} \rightarrow \kappa(y) l_{y}=l_{y}^{\prime}=\kappa(y) \exp \left[a \int_{\rho_{x y}} \phi_{\mu}^{\prime} d x^{\mu}\right] \kappa^{-1}(x) l_{x}^{\prime}=\exp \left[\int_{\rho_{x y}}\left(a \phi_{\mu}^{\prime}+\kappa^{-1} \kappa_{\mu}\right) d x^{\mu}\right] l_{x}^{\prime}
$$

where $\mu$ denotes the derivative with respect to $x^{\mu}$. A gauge transformation defined by (2), contains a path-dependent part, a functional, defined by (1) termed the essential gauge, and a point function $\kappa(x)$ referred to as the assigned gauge. The gauge transformations are at times introduced differently in literature. Weyl himself had introduced them as the length recaliberations and the 
parallel transport rule arose out of a resulting necessity to define a torsion free derivative. However, the metric and the affine connections that define the parallel transport, are independent concepts and both are taken into account in all formulations. This and the other rether presentations are equivalent to the present one; the ptresent one is better suited for the present purpose.

\section{Quantum mechanics}

\section{Standard formulation}

Standard formulation of quantum mechanics is available in the text books in detail. Here we discuss a few of the postulates for their relevance to the other formulations discussed here. The state of a physical system is described completely by its wave function, or the amplitude $\psi=\psi(\mathbf{r})$. If there are two possible states $\psi_{1}$ and $\psi_{2}$ for a system, then their linear combinations $c_{1} \psi_{1}+c_{2} \psi_{2}$ are also possible states; this is known as the Superposition Principle. Probability of a particle being in the space region between $\mathbf{r}$ and $\mathbf{r}+d \mathbf{r}$ is proportional to $|\psi(\mathbf{r})|^{2} d \mathbf{r}$, which is known as the Born Rule. Physical observables are represented by mathematical operators. If a state $\psi$ of a system is a linear combination $\psi=c_{1} \psi_{1}+c_{2} \psi_{2}$ of two eigenstates $\psi_{1}$ and $\psi_{2}$ of an observable $A$, then the outcome of a single measurement is

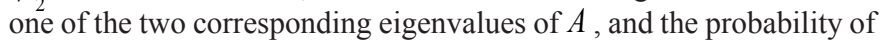
it being the eigenvalue corresponding to $\psi_{n}$ is equal to $\left|c_{n}\right|^{2}, n=1,2$ . This constitutes the basis of the Copenhagen Interpretation, i.e., upon measurement the wave function collapses in a single state. Representation of most of the observables can be determined from that of the momentum $p$, which has a concrete representation by a differential operator, which is, $p=-i \partial=-i \partial / \partial x$, where $x$ is the position. Two canonically conjugate observables, e.g., the position and momentum, are assumed to satisfy a commutation relation. These yields the Uncertainty Principle: There is a limit to the accuracy of two conjugate observables in their simultaneous measurement. These postulates differ fundamentally from the classical formulation where the physical observables completely describe the state of a system that is precisely determined. Evolution of a system is described by the Schrödinger equation.

$$
i \frac{\partial \psi}{\partial t}=H \psi
$$

Where $H$ is the Hamiltonian operator obtained from its classical form by replacing its argument observables by their operator representatives. Here $t$ is the time treated as a parameter and (3) refers to the one-dimensional non-relativistic case. Generalization to the Riemannian case, which includes the relativistic case, entails in letting $p^{\mu} \rightarrow i \partial ; \mu$, where $p^{\mu}$ and $\partial ; \mu$ is the contra variant components of the momentum and the covariant derivative. In the relativistic formulation, time loses its independent parameter character and in general Riemannian spaces; it is replaced by arc length as will be discussed.

\section{Feynman's path integral formulation}

In Feynman's path integral formulation of quantum mechanics (Section 4.3), a particle is assumed to take all alternative paths available to it and the wave function is defined as an aggregate of the phase factors associated with infinity of trajectories. Quantum mechanical amplitude $K\left(x, x_{0}\right)$ for a particle to go from $x_{0}$ to $x$ is defined to be the equiweighted sum of the phase factors $\exp \{i S[\rho(t)]\}$ along all paths from $\left[x_{0}, t\left(x_{0}\right)\right]$ to $[x, t(x)]$, where $S[\rho(t)]$ is the classical action along a trajectory $\rho(t)$ joining these two points, i.e.,

$$
K\left[x, t(x) ; x_{0}, t\left(x_{0}\right)\right]=\sum_{\text {all paths }} w(\rho) \exp \{i S[\rho(t)]\} .
$$

The weight $w(\rho)$ is equal for all trajectories. The amplitude admits the following integral representation:

$$
K\left[x, t+\varepsilon ; x_{0}, t\left(x_{0}\right)\right]=\int d y v(y) \exp (i S[x, t+\varepsilon ; y, t]) K\left[y, t ; x_{0}, t\left(x_{0}\right)\right]
$$

Where $v(y)$ is a suitable measure and $S[x, t+\varepsilon ; y, t]$ is the action along the classical trajectory from $(y, t)$ to $(x, t+\varepsilon)$. Letting $\left(x_{0}, t_{0}\right)$ vary over the entire extended manifold yields the following representation of the wave function:

$$
\psi(x, t+\varepsilon)=\int d y v(y) \exp (i S[x, t+\varepsilon ; y, t]) \psi(y, t) .
$$

The differential equation for $\psi$ is obtained by expanding both sides of (6) in powers of $\varepsilon$ and by term by term comparison; only a few terms in the expansion are needed. Feynman had initially obtained the Schrödinger equation. Since then, the Klein-Gordon equation in the electromagnetic field (Section 4.4.2) and generalized to the Riemann spaces has also been obtained (Section 4.4.3). The Dirac equation created some complications. However, spinors have been formulated to be the solutions of the generalized Klein-Gordon equation with some improvements over the Dirac equation. ${ }^{13}$

Feynman's formulation has a clearer conceptual basis, which is basically a pure particle formulation but not entirely as a wavelike coherence on the trajectories is introduced in the definition of the wave function. As a result, the wave function appears somewhat of a superposition of infinitely many waves. The formulation yields useful methods to deduce various quantum mechanical results and it modifies the dynamical equations in curved spaces by a curvature term, which is known to be physically significant. Extension to the curved Riemannian spaces involves some mathematical complexities but the resulting calculus is still quite straightforward (Sections 4.3 and 4.4.3). Technically, the path integrals on such spaces are constructed by patching infinitesimal trajectories in the tangent spaces. This construction results in replacing $d y$ with the invariant measure $\sqrt{|\tilde{g}(y)|} d y$ with $|\tilde{g}(y)|$ being the determinant of the metric $\tilde{g}(y)$.

\section{Wheeler's wiener integral in weyl's geometry}

In the gauge transformations (Section 3.1), Weyl identified $\phi_{\mu}^{\prime}$ with $\phi_{\mu}$, where $\phi_{\mu}$ are the electromagnetic potentials, and took $a$ to be a real constant. Later London ${ }^{7}$ showed that with $a=-i e$ and $y$ varying along the classical trajectory of a particle of charge $e, l$ is directly proportional to an extended de Broglie wave function associated with the particle. The Weyl-London gauge transformation is widely used in quantum mechanics in its original as well as generalized to multidimensional cases. Further significant progress was made by formulating and extending Hamilton's action principle in the framework of gauge transformations with $a \phi_{\mu}^{\prime}=-i e S_{\mu}$, where $S$ is the classical action, for a charged particle in an electromagnetic field. ${ }^{8}$ This formulation was later extended to include the multidimensional gauge fields. ${ }^{9}$ Original formulations have undergone through some further adjustments [Sec. 2.3.3]. In the meantime, Wheeler investigated the correspondence between the Weyl geometry and quantum mechanics in some detail. ${ }^{10}$

For lack of a unique trajectory, Wheeler assigned infinity of trajectories and defined the Wiener integral representations of the amplitude, $\psi^{+}$and its conjugate $\psi^{-}$with the associated measurable 
probability density $\psi^{+} \psi^{-}$. Specializing the formulation for a charged particle in an electromagnetic field in its non relativistic approximation, the probability density was shown to satisfy the Fokker-Planck, diffusion equation, which had been used before to interpret the Schrödinger equation as a diffusion phenomenon. ${ }^{11}$ This associates Wheeler's formulation with quantum mechanics albeit in a limited way.

While Feynman's formulation has been quite successful in deducing the quantum mechanical equations, Wheeler's, has not been. Also, this formulation suffers from an encumbrance of somewhat arbitrary, intricate and not all that tenable reasoning underlying its assumptions. Therefore, Wheeler's formulation will not be discussed futher here. It is mentioned above mainly for its historical significance and for its novel approach.

\section{Formulation based on action principle}

In this section, we describe a recently developed path integral formulation based on an extention of Hamilton's action principle in the framework of gauge transformations (Section 1.1), referred to as the "Geometrical Formulation" (Section 4.3.3) .

\section{Extended action principle}

Hamilton's action principle assumes the action $S$ to be stationary about the particle path $\rho_{x y}$, i.e., $\rho_{x y}$ is an extremal defined by this variational characterization. The principle is stated as

$$
\delta S=\delta \int_{\rho_{x y}} d S=\int_{\rho_{x y}}^{c, \text { inf }} d S=S\left(\rho_{x y}^{c, \text { inf }}\right) \cong 0
$$

where $\cong$ indicates that the equality holds up to the first order in the area enclosed by each closed curve $\rho_{x y}^{c \text { inf }}$ obtained as the union of an arbitrary curve $\rho_{x z y}$ in a small neighborhood of $\rho_{x y}$ and another arbitrary curve $\rho_{y z^{\prime} x}$ in a small neighborhood of $\rho_{y x}$ with $x$ and $y$ fixed. Now, the classical action principle can be expressed as

$$
1+a S\left(\rho_{x y}^{c, \text { inf }}\right) \cong \exp \left[a S\left(\rho_{x y}^{c, \text { inf }}\right)\right] \cong 1
$$

for all closed curves $\rho_{x y}^{c \text {,inf }}$ enclosing infinitesimally small areas with an arbitrary nonzero constant $a$. From (2), the choice $\phi_{\mu}^{\prime}=a S_{\mu}$ defines the gauge group element associated with $\rho_{x y}^{c, \text { inf }}$ to be $\exp \left[a S\left(\rho_{x y}^{c \text {,inf }}\right)\right]$.

Thus, (7) formulates the classical action principle in terms of the gauge transformations. Classical characterization of the particle motion stated in (7) admits a natural extension:

$$
\kappa(y) \exp \left[a S\left(\rho_{x y}\right)\right] \kappa^{-1}(x)=1,
$$

which is a founding assumption of this formulation. The left side of (8) is the gauge group element with $\phi_{\mu}^{\prime}=S_{\mu}$, associated with an arbitrary curve $\rho_{x y}$, closed or unclosed, and the equality is assumed to hold exactly. For the curves $\rho_{x y}^{c, \text { inf }},(8)$ reduces to (7) up to the first order as an extension should. Infinitely many solutions of (8) facilitating the passage of a particle will be called the physical paths.

The assigned gauges in the Weyl geometry are arbitrary. We require that $\kappa(x)=\kappa\left(x^{\prime}\right)$ if and only if the points $x$ and $x^{\prime}$ in the underlying manifold are physically equivalent, constituting another founding assumption. For illustration, consider a free particle collimated by a small aperture and detected at some distance. The particle experiences the same physical conditions at all points in the interior of any path it may take and thus at all points except about the aperture and the detection point. However, it is subjected to different interactions at the aperture and at the detector, which are also geometrically localized and separated from the other points. Thus, the aperture and detection point are both physically different from the other points and from each other. Therefore, the assigned gauges are different at the aperture, at the detector and at the other points of the relevant region where they are equal to a constant. Present assumptions about the assigned gauges thus, take the experimental configuration and the involved interactions into account.

Disctinction between the assigned and the essential gauges is not so clear in literature, where they are considered essentially inconsequential by requiring meaningful physical quantities to be gauge invariant. In the present formulation, they assume a significant role as they are defined in terms of the physical conditions relevant to the physical system including the method of observation.

From (8), a constant value of the assigned gauges cancels out for a physical system confined to a region with all of its points being equivalent to each other, e.g., a free particle. In case of the closed curves as the action principle stated in (7) and the Bohr orbits,, the assigned gauges are ineffective, again due to cancelations. In all such cases, (8) reduces to $\exp \left[a S\left(\rho_{x y}\right)\right]=1$. Since nonzero real values of the action for such particle trajectories cannot be excluded, $a$ should be purely imaginary, which can be set equal to $i$. Thus, London's assumption of $a$ being purely imaginary, ${ }^{7}$ is deduced here as a result. Since $a$ is the same constant for all cases, (8) reduces to

$$
\kappa(y) \exp \left[i S\left(\rho_{x y}\right)\right] \kappa^{-1}(x)=1 .
$$

Left side of (8) is the length acquired by a unit vector transported along $\rho_{x y}$. Thus, for a trajectory to be physical, i.e., an allowed particle path, (8) requires a vector attached to the particle to regain its length at some point along the path. Then it follows from (9) that the vector regains its length along such a path periodically.

It is clear from (8) and (9) that the assigned gauges participate in describing the observable effects.

\section{Physical trajectories}

Since there are no preferred physical paths, all are equally likely and a particle trajectory would be determined by random selection. Thus, while a particle follows a definite trajectory, it is not determinable due to the randomness.

If $\rho_{x y}$ and $\rho_{y z}$ are two physical paths, then the union $\rho_{x z}$ of $\rho_{x y}$ and $\rho_{y z}$ is also physical, termed a continuing union. The physical trajectories can be classified under two categories: Monotonic and nonmonotonic. Along a monotonic trajectory, the action increases (decreases) monotonically as the path is traversed. If a trajectory is not monotonic, it is nonmonotonic. Nontrivial monotonic physical paths with smallest allowed value of the action in magnitude will be called the elemental physical paths. For a constant $\kappa$, the elemental physical paths $\rho_{x y}$ are defined by $S\left(\rho_{x y}\right)= \pm 2 \pi$. Thus, for a monotonic trajectory, a particle travels along a randomly selected elemental from one point to the other and then along another elemental from its arrival point to the next. It follows that a general monotonic particle trajectory is constituted of a sequence of randomly selected elementals. Nonmonotonic physical trajectories can be considered the continuing unions of their monotonic segments. 
A particle originating at a source and detected some distance away, would travel along a continuing union of randomly selected elementals, which may or may not coincide with or be close to the extremal of the classical action principle. Thus, the particle would be detected as a localized entity, i.e., as a particle, but not necessarily at the location determined by the action principle. If such an experiment is conducted with a large collection of identical particles or many times, each with a single particle with particles in all repeats being identical, then each particle would travel along an independent continuing union of the elementals. The collection of the detected particles may or may not cluster about the classically determined location. This agrees with the observations.

For a macroscopic system, that of a classical scale, the trajectories available to a particle have been shown to be concentrated about an extremal. The basic argument is that there are many equiaction trajectories in a second order neighborhood of a classical trajectory because of a second order difference in the action along the trajectories in its neighborhood. Such trajecories in pairs can be shown to constitute non-monotonic physical trajectories. Thus, each monotonic segment of each such trajectory can facilitate passage of a particle. Consequently, a particle would travel along an almost classical trajectory. This implies also that the major part of an expected observation can be gleaned by studying the classical trajectories.

The argument applies and the conclusion holds equally well for the monotonic segments of a classical magnitude of the nonmonotonic physical trajectories that are the continuing unions of their piecewise monotonic segments. The equiaction trajectories can be shown to exist also in a small neghborhood of a nonclassical trajectory but spread over a first order neighborhood with consequent reduced density.

\section{Role of gauge in observations}

Consider the double slit experiment where identically prepared particles, e.g., the electrons, encounter two slits, at $x_{i}$ and $x_{f}$, in a screen, and observed at an arbitrary location $\hat{x}$ on an observation screen. A pair of trajectories meeting at $\hat{x}$ can be considered a continuing union of two monotonic trajectories $\rho_{x_{i} \hat{x}}$ and $\rho_{\hat{x} x_{f}}$ each of a classical extent constituting a nonmonotonic trajectory $\rho_{x_{i} \hat{x} x_{f}}$

from $x_{i}$ to $\hat{x}$ to $x_{f}$. In this arrangement, the assigned gauge at $\hat{x}$ cancels out reducing (9) to

$$
\kappa\left(x_{f}\right) \exp \left\{i\left[S\left(\rho_{x_{i} \hat{x}}\right)-S\left(\rho_{x_{f} \hat{x}}\right)\right]\right\} \kappa^{-1}\left(x_{i}\right)=1 .
$$

For identiacal slits, the points $x_{i}$ and $x_{f}$ are physically equivalent implying that $\kappa\left(x_{i}\right)=\kappa\left(x_{f}\right)$ and hence, the solutions of (10) are given by

$$
S=S\left(\rho_{x_{i} \hat{x} x_{f}}\right)=S\left(\rho_{x_{i} \hat{x}}\right)-S\left(\rho_{x_{f} \hat{x}}\right)=2 n \pi
$$

with $n$ being an arbitrary integer. An extremal joining any two points in this case is a straight line with the momentum $p$ of the particle(s) being almost constant. Thus, if the monotonic segments $\rho_{x_{i} \hat{x}}, \rho_{\hat{x} x_{f}}$ of the corresponding nonmonotonic trajectory $\rho_{x_{i} \hat{x}_{f}}$ are extremals of the action principle, then $S=p \Delta r^{\prime}$, where $\Delta r^{\prime}$ is equal to the difference between the path lengths of the straight lines from $x_{i}$ to $\hat{x}$ and $x_{f}$ to $\hat{x}$. It follows that for a union of two straight lines transmitting a particle of momentum $p$ to be physical, it is required that $\Delta r^{\prime}=2 n \pi / p=\Delta r_{n}$. Since the physical trajectories are concentrated about the extremals $\rho_{x_{j} \hat{x}}$ and $\rho_{\hat{x} x_{f}}$, as explained in Sec. 4.4.2, a high concentration of the physical trajectories and hence of the particles would exist in small neighborhoods of such points on the observation screen, denoted by $\hat{x}_{n}$, decreasing away from them. In between the maxima, particles can only reach along nonextremals with small associated density due to a smaller concentration of the physical trajectories there. The particle density distribution deduced above resembles the intensity distribution resulting from the interference of two identical wavelets emanating from $x_{i}$ and $x_{f}$ as observed.

The above observation underlies the doctrene of the wave-particle duality, which does not make a distinction between the particle density distribution and the intensity pattern produced by the interfering waves, rendering the argument underlying this doctrene rather weak. In the geometrical formulation, the particle density distrbution is obtained above from the defintion of the physical trajectories.

If an intrusive observation is made behind a slit, e.g., by scattering the photons from the particle beam, path of the beam is altered which would shift the location of the corresponding beam on the observation screen. This shift can be calculated precisely for the classical trajectories, which is indicated above to yield a close approximation to the expected observation. Estimates (Section 4.4.1) show that for small values of the shift $S$, the particle beams can still be considered almost correlated since there would still be many pairs of almost extremals with a common point on the observation screen in the small neighborhoods of $\hat{x}_{n}$. This implies the existence of a shifted and smeared density pattern. As the scattering effects increase, the shift increases shifting and smearing the density distribution further. When the shift increases still further, the correlation between the beams is almost completely lost. Consequently, $\kappa(\hat{x})$ cancels out for almost no trajectories invalidating (10). Instead, the beams travel independently, which are almost classical particle trajectories as discussed in Section 4.4.2.

It can be inferred from the above discussion that a set of trajectories transmitting the particles to an observation region would produce a density distribution resembling an interference pattern if and only if the net gauge effect is insufficient to enable an extraction of "which path" information. If "which path" information is available at the terminal point $\hat{x}$ of a trajectory, it cannot be common to the corresponding pairs of the beams.

The particle behavior here is described in terms of the correlation of trajectories, which is a geometrical concept, in contrast with the entangled physical systems, or particles, in standard quantum mechanics. In the above example of the double slit experiment, assigned gauges make significant contribution to the observation. Essentail gauges also impact upon the observations as in the AharonovBohm effect, predicted by Bohm ${ }^{12}$, which has been experimentally verified. ${ }^{14,15}$

To describe the Aharonov-Bohm effect, consider an experimental set up in which a controlled magnetic field is generated by a long vertical solenoid by controlling the current through its coil mimicking a magnetic monopole. An electron beam is split in two at a point $x_{i}$ . One of the beams travels along a trajectory $\rho_{1}$ on one side of the cylinder and the other along $\rho_{2}$ on the opposite side, both shielded from the magnetic field. Then the beams meet at another point $\hat{x}$ . Thus, the union $\hat{\rho}$ of $\rho_{1}$ and $\rho_{2}$ encloses the magnetic field but neither of the beams passes through it. Since the potentials were considered only the mathematical auxiliaries and the observable effects were still assumed to be produced by the fields, the AharonovBohm effect was considered an anomalous behavior predicted by quantum mechanics. 
In the above arrangement, the assigned gauges can be seen to become ineffective. As in case of the double slit arrangement with no gauge effect, where the physical trajectories are given in (10), the physical trajectories for the present arrangement are given by $S=2 n \pi$ , where

$$
S=S\left(\rho_{1}\right)-S\left(\rho_{2}\right)=S_{0}+e \int_{\hat{\rho}} \phi_{\mu} d x^{\mu}=S_{0}+e f .
$$

with $S_{0}$ being the free particle part of the action. Again as in case of the double slit arrangement, this produces a particle density distribution resembling the interference pattern. As $f$ is varied, it follows from (11) that the density pattern shifts repeating itself with period $2 \pi / e$ with respect to $f$. This agrees with the observations.

This effect was predicted ${ }^{12}$ based on the soluions of the Schrödinger equation but the argument is valid for the Klein-Gordon equation also. Since the underlying manifold in this case is multiply connected, the wave functions on one sheet differs from that on the other by a phase unless $f=2 \pi / e$ resulting in a constructive interference and the periodicity; elsewhere the density pattern resembles the interference pattern of the two interfering waves differing by a nonzero phase fifference modulo $2 \pi$. Thus, in the geometrical formulation, the Aharonov-Bohm effect is described solely by the definition of the physical trajectories in a sharp contrast with the standard argument based on the interfering waves.

The double-slit experiment and the Aharonov-Bohm effect share an essential similarity. In both of the cases, the observed effect depends on the fact that the two beams are related with each other through a gauge factor resulting from an alteration to the underlying geometry. In the former, this alteration results from the two slits generating an assigned gauge distribution and in the later, from a hole punctured by the field confined almost to a point, generating an essential gauge factor. In any case, the gauge factors, both assigned and essential, participate in producing the observable effects. Details of the above including the estimates are available elsewhere (Sections 4.4.1 and 4.4.2).

\section{Extended path integral formulation}

With $a \phi_{\mu}^{\prime}=i S_{\mu}$ and constant gauge, for a unit vector, i.e., $l_{x}=1$, $l_{y}^{\prime}$ in (2) is a typical phase factor term $\exp \{i S[\rho(\tau)]\}$ in Feynman's representation of the amplitude, or wavefunction (Section 4.2). In view of this observation, Feynman's quantum mechanical amplitude for a particle to go from $\left[x_{0}, \tau\left(x_{0}\right)\right]$ to $[x, \tau(x)]$ is naturally adjusted to read

$$
\begin{aligned}
K\left\{\kappa ;[x, \tau(x)],\left[x_{0}, \tau\left(x_{0}\right)\right]\right\} & =\sum_{\text {all paths }} w(\rho) \kappa(x) \exp \{i S[\rho(\tau)]\} \kappa^{-1}\left(x_{0}\right) \\
& \text { from } x_{0} \text { to } x
\end{aligned}
$$

which is just a slightly adjusted form of Feynman's formulation but with a significant difference in that it includes the assigned gauges that incorporate the impact of an observation on the system as long as an intrusion altering the assigned gauge distribution is not strong enough to decohere the system of trajectories. If not, the representation of (12) remains valid in each region where the stated condition is satisfied and (12) would yield a different amplitude in each such region. Thus, the present formulation improves significantly over the others.

Since the wavefunction is defined in (12) with $x_{0}$ being arbitrary, it defines the amplitude or wavefunction as an aggregate of the Weyl lengths at $[x, \tau(x)]$ of a unit vector transported to this point along all trajectories from everywhere, defined in (2) with $a \phi_{\mu}^{\prime}=i S_{\mu}$, providing a geomerical interpretation of the wavefunction. Following the standard procedure, (12) is expressed as

$$
\kappa^{-1}(x) K^{\prime}(\kappa ; x, \tau+\varepsilon)=\int d \hat{m}(y) \exp [i \hat{S}(x, \tau+\varepsilon ; y, \tau)] \kappa^{-1}(y) K^{\prime}(\kappa ; y, \tau) ;
$$

where $K^{\prime}\left(\kappa ; x^{\prime}, \tau^{\prime}\right) \equiv K\left\{\kappa ;\left[x^{\prime}, \tau^{\prime}\right],\left[x_{0}, \tau\left(x_{0}\right)\right]\right\} \quad$ and $\quad \hat{m}(y) \quad$ is a suitable measure. This yields the following representation of the wavefunction:

$$
\kappa^{-1}(x) \psi(\kappa ; x, \tau+\varepsilon)=\int d \hat{m}(y) \exp [i \hat{S}(x, \tau+\varepsilon ; y, \tau)] \kappa^{-1}(y) \psi(\kappa ; y, \tau) .
$$

For a constant gauge (14) reduces to Feynman's representation of the quantum mechanical wavefunction $\psi$ given by (6), which is the same as (14) with $\psi$ replacing $\kappa^{-1} \psi(\kappa)$ implying that $\kappa^{-1} \psi(\kappa)=\psi$ . The wavefunction $\psi(\kappa)$ representing the basic physical system together with the impact of the observing system can be constructed by multiplying $\psi$ by the applicable assigned gauge. As discussed in Section 4.4.3., close approximations to the assigned gauges can be obtained for a given physical sytem, which can be improved further. It is clear that the wavefunction and the characterization of the physical trajectories given in (9) together describe the motion more completely than either one alone. This adds a significant bit to the prevailing formulations of quantum mechanics.

In the geometrical formulation, an act of observation assigns certain gauges to points in the underlying manifold. Thus, making a measurement on $\psi$ alters it to $\psi(\kappa)$, which depends on the observing system and the interactions involved. Clearly, the wavefunction can be altered by altering the details of an observation. Thus, the concept of an unambiguous measurement cannot be assigned to a wavefunction. To eliminate the ambiguity, an associated gauge invariant quantity should be constructed and measured for a physical measurement to be objective and meaningful. Wheeler constructed the conjugates of tensors in Weyl's geometry with the product of a tensor and its conjugate being a quantity of vanishing Weyl weight, which was arbitrarily assumed to be physically measurable.

In the present formulation, it can be concluded that if a physical trajectory exists, then $|\kappa(x)|=|\kappa(y)|$ and since a constant factor in the assigned gauge cancels out $|\kappa(x)|=1$, implying that $\kappa(x)=\exp [-i \sigma(x)]$ with some $\sigma(x)$ for all $x$. This shows that the assigned gauges are reducible to phase factors. It follows that $\kappa^{-1}(x)=\kappa^{*}(x)$, the complex conjugate of $\kappa(x)$. Thus, $\psi^{*} \psi=(\kappa \psi)^{*}(\kappa \psi)$ is a real gauge invariant quantity, rendering $\psi^{*}$ a natural conjugate of $\psi$. Born's probability density $\psi^{*} \psi=|\psi|^{2}$ is clearly a uniquely defined gauge invariant quantity with a clear outcome of any measurement regardless of the gauge but at the expense of the observable information contained in the gauge factors, both essential and assigned.

\section{Quantum measurement}

There are several interpretations of quantum mechanics, each with its own explanation of the impact of method of observation, or the observer, on what is observed. Neither one of them has gained universal acceptance; each one has its supporters and critics with varied views, which have changed with time. The interpretations together with their critiques are described in detail in literature, which has grown into a massive body. Here we discuss a few major ones briefly, which have gained prominence from time to time and compare the understanding of the indicated phenomenon according to these interpretations with that in the geometrical formulation. For now, the considerations are restricted to the double slit experiment discussed in Section 4.4.3. 
The Copenhagen interpretation holds that the system is represented completely by a wavefunction, which collapses in one particular state consequent to a measurement, in the state that is observed. This view assigns dual nature, both wave and particle, to each of the entities, having no definite form until observed. Thus, the method of observation in a sense "bestows" the form upon the entity observed. In comparison, the geometrical formulation assumes a definite form, particle, for each of these entities and each one is expected to be observed as a particle individually. In a bulk, the entities' behavior in the gauge neutral case resembles that of a wave in the sense that the particle density distribution resembles the intensity distribution of two interfering wavelets. If the intrusion by the observing system is sufficiently strong to yield a definite outcome, it destroys the correlation between the trajectories from two slits breaking them up into more than one sets, one corresponding to the observation region and the others, covering its complementary region. The deduced observation then compares with a collection of classical particles in agreement with the observation. Separate wavefunctions can be constructed for each of the regions. Thus, the wavefunction $\psi$ representing the particles in the gauge neutral case "breaks up" into more than one fragments. The fragment corresponding to the observation region can be considered the "collapsed" form of $\psi$ providing an interpretation of the "wavefunction collapse."

The Bohm formulation ${ }^{16}$ or the pilot wave interpretation initially proposed by de Broglie, assumes that these entities are particles and each particle follows a definite trajectory but guided by a wave determined by the state of system. Thus, a particle passes through one of the slits but the wave passes through both and the probability of a particle being at a location on the observation screen is proportional to the resultant intensity of the interfering pilot wavelets at that location. Thus, the particle density pattern resembles the intensity distribution of two interfering wavelets without the particles assuming a wave form. Particle trajectories remain unknown, which are considered the hidden variables. Concerning the collapse of a wavefunction, the Bohm theory considers a universal wavefunction incorporating the observed and the observing systems together as a composite system, which never collapses; collapse occurs only in the phenominological sense applicable to a subsystem, which evolves separately caused by the decoherence resulting from an intrusion by a measurement.

According to the geometrical formulation, each one of the entities, assumed to be a particle, passes through one of the slits, which remains unknown but due to randomness. Also, while a definite trajectory cannot be assigned to a particle, sets of the physical trajectories can be computed from the information about the observed and the observing systems and thus, they are not completely hidden. The probability of finding a particle at a location is proportional to the density of the paths available for its passage, which for the identical slits and weak intrusions is shown to resemble the intensity distribution of two interfering wavelets originating at the slits. Two systems, the observed and the observing one, are considered together to determine the assigned gauges, which for strong intrusions, "decohere" the sets of trajectories. This decoherence causes the wavefunction to decompose into fragments, the observed one of them being the "collapsed" wavefunction, as discussed above. Wavefunction of the composite system, the observed and the observing one together, does not enter the considerations. Impact of the observing system is incorporated in the wavefunction through the assigned gauges.

Many worlds view or the relative state formulation ${ }^{17}$ assigns purely wave nature to these entities. It was developed by coupling the observing system with the observed one through wavefunction of the composite system. Each measurement leaves each subsystem in a relative state with respect to the other. There is no quantification of such alterations. With each observation, system's state branches off to different non-communicating worlds, each one corresponding to each of the possible outcomes of an observation. What is observed depends on the world the observer enters. Such branching off occurs at each subsequent observation. Thus, the outcome of a measurement depends on all previous measurements.

In the extended formulation, the method of observation couples the two systems through the assigned gauges. Basic physical system and the assigned gauges determine the observation. Each act of observation assigns a computable multiplicative gauge factor altering the "state" of the observed system, which is altered further by each subsequent measurement. If an observation causes a strong intrusion, the system of trajectories changes in a drastic way. As discussed above, one "branch" of the trajectories corresponds to the observation in the lab and the other, to its complement but in the same "world," not in a different one, and what is observed depends on which branch one observes. Each one of these branches has an associated wavefunction. Such "branching" off occurs with each measurement yielding a definite outcome. This provides an interpretation of the "many worlds" view.

The concept of a wave is basic to the interpretations of quantum mechanics discussed here, which is irrelevant to the present formulation. The concept of gauge transformations does not enter the formulations and interpretations of quantum mechanics, which is fundamental to the geometrical formulation. Thus, the geometrical formulation differs radically from the standard quantum mechanics and its interpretations in its underpinnings as well as implications, although it has parallels.

In the framework of the geometrical formulation, the expected observation in the experiments involving large number of particles can be determined to a large extent, with the usual information about the observed and the observing systems prior to making a measurement, distinguishing it fundamentally from the interpretations as they do not have such predictive ability; outcome of an observation is known only after it has occurred; they only attempt to describe how the observation ocurred.

\section{Concluding remarks}

Present paper presents a brief review of the earlier formulations of quantum mechanics and a more recent, geometrical formulation, together with their critical comparative study. An extension of Hamilton's action principle in the framework of Weyl's gauge transformations constitutes the basic founding element of the geometrical formulation. Consequent clear charcterization of the particle trajectories augments the standard formulations and interpretations of quantum mechanics. The fundamental underlying principles of the geometrical formulation, stated in (7) and (8) of the classical and quantum mechanics, respectively, show that they are more closely related to each other than known previously and both are intertwined with the gauge transformations.Futher, the bserved impact of the method of a physical measurement on its outcome is formulated in terms of the assigned gauges determined by the configuration of the observing system and the interactions between the observed and observing systems. Thus, the assigned gauges that were largely ignored, wasted, in earlier related studies are used effectively in the geometrical formulation to develop a view of quantum measurement that differs radically from the views in the prevailing interpretations of 
quantum mechanics. This provides a novel and clearer understanding of the quantum measurement, which greatly improves upon the earlier explanations. The geometrically based theory remains indeterministic and nonlocal, which appear to be the facts of nature as supported by the observations, but with greatly improved understanding of the indeterministic and nonlocal attributes of physical phenomena.

In the geometrical formulation, the extended wavefunction admits an interpretation as an aggregate of the lengths acquired by a unit vector transported from everywhere along a collection of trajectories providing a clearer geometrical view of the wavefunction. Impact of a measurement on the wavefunction is shown to be directly related to its impact on the physical trajectories adding to the clarity of the process of physical measurements. The geometrical formulation renders some justification to Born's probability postulate also, which was a founding postulate in the other formulations. The wavefunction and the probability density supplemented with the physical trajectories (Sections 4.4.1 and 4.4.2) describe the behavior of a system more completely than otherwise. This constitutes a significant addition to the previling formulations of quantum mechanics. This formulation also enables one to determine the expected outcome of an observation from the information about the systems, the observing and observed, albeit to a limited extent. Other formulations and interpretations of quantum mechanics do not have such predictive ability; they only "explain" the observation if it is known.

Comparative study of the foumulations and interpretations of quantum mechanics in this paper indicates that the geometrical formulation improves significantly upon the earlier ones and its further studies are likely to provide clearer undertanding of the "mysteries" of quantum mechanics.

There are issues with the prevailing formulation of quantum fields also, which have been omitted in the present paper. Recent developments addressing such matters are available elsewhere. ${ }^{3}$ In this recent formulation, which is more streamlined, quantization is deduced from the periodicity of phase factors appearing in (4) and (12) providing a unified framework for somewhat disjointed theories. A cosequent result of major significance is elimination of unphysical infinite vacuum energy in contradistinction with the prevailing second quantization. A novel interpretation of time results in the process.

\section{Acknowledgments}

None.

\section{Conflicts of interest}

The author declares there is no conflcit of interest.

\section{References}

1. Feynman RP, Hibbs AR. Quantum Mechanics and Path Integrals. McGraw-Hill, New York, USA. 1965. p. 1-21.

2. Kleinert H. Path Integrals in Quantum Mechanics, Statistics, Polymer Physics, and Financial Markets. 5th edn. World Scientific, Singapore. 2009.

3. Shiv Vatsya. Fundamentals of Quantum Theory. 2017.

4. Schulman LS. Techniques and Applications of Path Integration. Wiley, New York, USA. 1981

5. Kim YH, Rong Yu, et al. Delayed "Choice" Quantum Eraser. Physical Review Letters. 2010;84(1-5).

6. Weyl H. Space-Time-Matter. Chapters II, IV, Dover publication, New York, USA. 1951.

7. London F. Quantenmechanische Deutung die Theorie von Weyl. Zeitschrift für Physik A Hadrons and nuclei. 1927;42(5-6):375-389.

8. Vatsya SR. Gauge-theoretical origin of mechanics. Canadian Journal of Physics. 1989;67(6):634-637.

9. Vatsya SR. Mechanics of a particle in a gauge field. Canadian Journal of Physics. 1995;73(1-3):85-95.

10. Wheeler JT. Quantum measurement and geometry. Physical Review D. 1990;41(2):431-441

11. Nelson E. Quantum Fluctuations. Princeton University Press, Princeton, USA, 1987. p. 1-158.

12. Aharonov Y, Bohm D. Significance of Electromagnetic Potentials in the Quantum Theory. Physical Review. 1959;115(3):485-491.

13. SR Vatsya. Formulation of Spinors in Terms of Gauge Fields. Foundations of Physics. 2015;45(2):142-157.

14. RG Chambers. Shift of an Electron Interference Pattern by Enclosed Magnetic Flux. Physical Review Letters. 1960;5(1):3-5.

15. Tonomura A, Osakabe N, Matsuda T, et al. Evidence for AharonovBohm effect with magnetic field completely shielded from electron wave. Physical Review Letters. 1986;56(8):792-795.

16. Bohm D, Vigier JP. Model of the Causal Interpretation of Quantum Theory in Terms of a Fluid with Irregular Fluctuations. Physical Review. 1954;96(1):208-216.

17. Everett H. Relative State. Formulation of Quantum Mechanics. Reviews of Modern Physics. 1957;29(3):454-462. 